
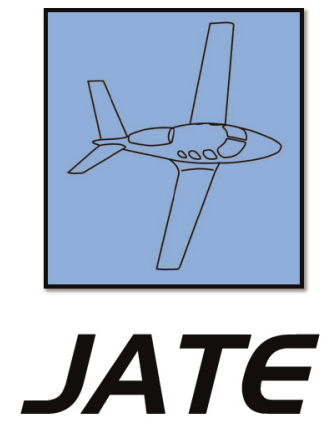

Journal of Aviation Technology and Engineering 2:1 (2012) 105-115

DOI: $10.5703 / 1288284314862$

\title{
A Linear Programming Model for Time-Alignment of Cockpit Voice Recorder and Flight Data Recorder Data
}

\author{
William A. Tuccio and Harold D. Townsend
}

Embry Riddle Aeronautical University

\begin{abstract}
Aircraft accident forensics include analyzing cockpit voice recorder (CVR) and flight data recorder (FDR) information. Each recorder is independent of the other and often share no common time frame. Analysts must align the two time scales using common events on each recorder, such as microphone keying events. Using test drive development and linear programming, we have created a two-step model to align the CVR and FDR recordings. The first linear model matches microphone keying events, while the second linear model optimizes alignment of the time scales. When the constrained optimization problem produces a feasible solution, a standardized computer-generated sentence is created that is useful for forensic reporting purposes.
\end{abstract}

Keywords: convolution, linear programming, recorder, time alignment, CVR, FDR

\section{Introduction}

Aircraft accident investigation is an essential process to determine probable cause and propose recommendations to reduce future risk. Investigators use many types of evidence to determine what caused an accident; one source of information is commonly known as the black boxes (Schueller, 1998). Commercial aircraft have two black boxes installed: a flight data recorder (FDR) and a cockpit voice recorder (CVR). The FDR records many aircraft parameters such as speed,

\footnotetext{
About the Authors

William A. Tuccio is a $\mathrm{PhD}$ student in the Aviation program with Embry-Riddle Aeronautical University. His experience and research interests are in the area of aviation, data-centric solutions, and transportation safety. Today, William contributes to transportation accident investigations. This work is the author's alone and does not reflect the official position of any employer or government agency.

Harold D. Townsend is a PhD student in the Aviation program with Embry-Riddle Aeronautical University, where he also acquired a Bachelor degree in Aeronautical Science later followed by an MBA from the University of Alaska Anchorage. His experience includes that of an airline pilot, aircraft mechanic, flight operations training manager, financial analyst and financial controller. His academic interest is airline finance and operations.
} 
altitude, and aircraft configuration. The CVR records pilot voice transmissions, cockpit noises, and engine sounds. Through a combination of the recorded information, investigators are able to analyze accident related events.

\section{Statement of Problem}

Forensic accident investigation presents many challenges. One critical challenge is an accurate and consistent time scale between different pieces of evidence. This arises when aligning the separate time scales of the aircraft CVR and FDR; Figure 1 shows the nature of the problem. The technologies used by many in-service CVRs are such that the audio recorded by the CVR lacks an external time reference. ${ }^{1}$ While FDRs often have an external time reference, for the purpose of this problem statement, it is sufficient to say that the FDR time reference is different from the CVR time reference (Gregor, 2006). Investigators successfully use a variety of techniques to align timing of CVR and FDR data, as well as timing of other data sources (i.e., air traffic control radar, witness statements, etc.).

\section{Statement of Purpose}

The purpose of this paper is to use software engineering test driven development (TDD) to demonstrate how linear programming can be applied to time synchronization between CVR and FDR data. After delimiting the problem, the paper develops and tests a linear programming model

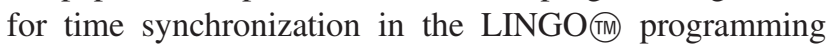
language.

\section{Nature of Problem}

A common technique used by accident investigators to align CVR and FDR data is to use pilot initiated radio transmissions as the basis of alignment (Gregor, 2006). That is, an analyst can hear when the cockpit microphone transmit button is depressed ("microphone keying") on the CVR, and can further bolster the accuracy of this observation by looking at an audio spectrum of the CVR content. The accuracy of microphone keying on the CVR can often be identified to within a tenth of a second. The FDR records many parameters-sometimes thousands of parameters-sampled at various rates varying generally from every four seconds to every 16th of a second, depending upon regulations and installation configuration (Federal Aviation Administration, 2010). One of the critical timing parameters recorded is microphone keying. Whenever a pilot keys the microphone, the FDR records the microphone keying as a binary value (i.e., 1 for keyed,

\footnotetext{
${ }^{1}$ State-of-the-art CVRs are beginning to employ frequency shift key (FSK) encoded data in CVR audio in accordance with internationally developed standards. However, many in-service CVRs lack this FSK component.
}

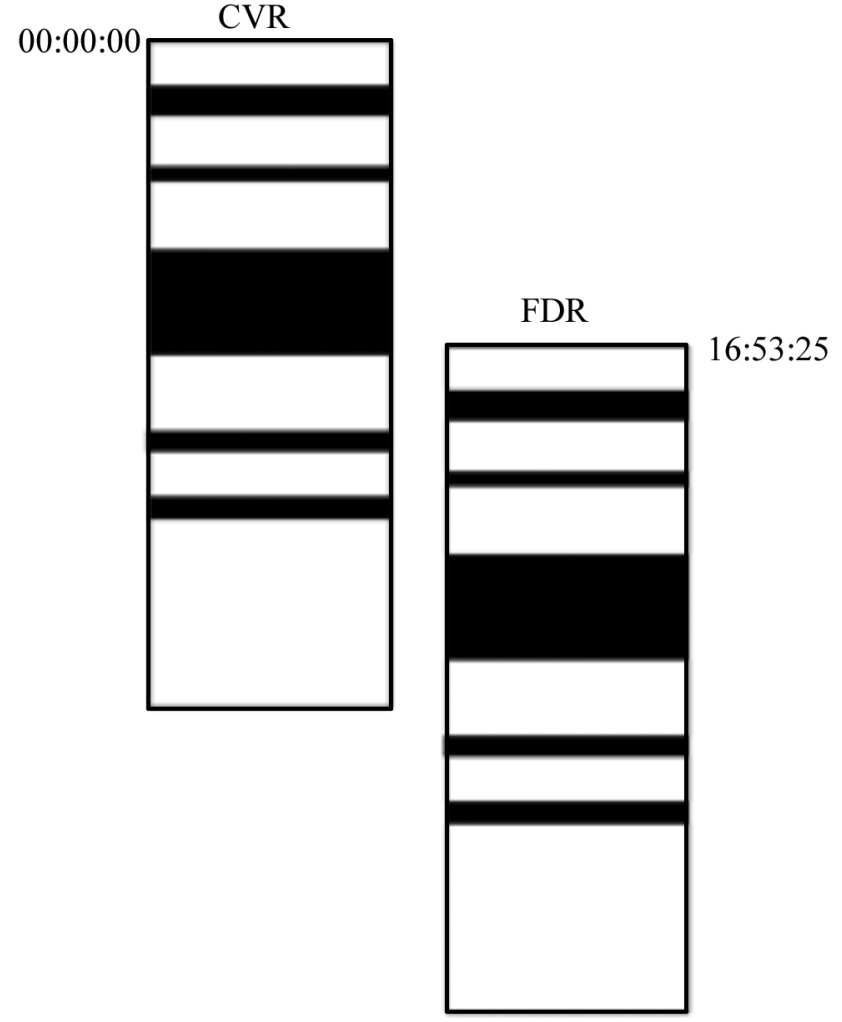

Figure 1. Time scale alignment issue; black shading indicates events common to the CVR and FDR.

0 for not keyed). Microphone keying is most often sampled every one second. This record of keying in the CVR and FDR forms the basis of timeline alignment.

The CVR microphone keying identification accuracy and the FDR microphone keying sample rate pose an optimization problem for the synchronization of the timelines. The problem can be stated as an optimization of two or more series of chronological events where the events are sampled with different accuracies or rates.

The problem can be further generalized by considering additional realities of recording devices and the forensic environment. One reality is the accuracy of the device recording and playback rate ("timebase"). That is, a 60minute period may be recorded in 58 minutes or 62 minutes, or more generally a tolerance of $\pm r$, where $r$ is the tolerance of recording timebase. Further, $r$ could be a variable, influenced by power fluctuations or environmental acceleration factors that may be experienced in an accident. While $r$ was a significant factor in tape based, legacy recorders, today's solid-state recorders have $r^{\prime}$ s with small variations over time. For example, US regulations require a tolerance of $\pm 0.125 \%$ per hour, (Federal Aviation Administration, 2010). This equates to \pm .75 seconds for a 10 minute recording or \pm 6.75 seconds for a 90 minute recording.

Another reality of the forensic environment is that CVR and FDR microphone keyings may be mixed with other events that have greater accuracy or forensic importance. 
For example, a critical warning may be recorded by the CVR and FDR and be added to the event timeline. Further, there may be missing events between the two timelines, for example, a short microphone keying could be heard on the CVR that was not sampled by the FDR (i.e., for microphone keyings with a total duration of less than one second).

\section{Literature Review}

Combining the CVR and FDR timescales is an essential step in the investigation process. The procedure is most often accomplished by an investigator analyzing the audio of the CVR for the start and stop of radio transmissions and recording this numerical information. The series of transmissions are then compared to a series of microphone keyings recorded on the FDR. These events can then be correlated to other time series events, such as air traffic control. (Brazy, 2009; National Transportation Safety Board, 2010; O'Callaghan, 2009; Smith, 2009). Text summarizing the analysis is often presented as follows:

The times reported in the attached CVR transcript are Eastern Standard Time (EST), and represent the time that each comment or sound begins. Time is specified to the nearest whole second, unless otherwise noted.

The CVR and FDR data were synchronized to one another by comparing the FDR "Key VHF" parameter with radio transmissions as heard on the CVR recording. By comparing the CVR elapsed time (time since the beginning of the CVR recording) for radio transmissions to the corresponding FDR Subframe Reference Number (SRN) for "Key VHF," a relationship between the CVR elapsed time and the FDR SRN time can be developed.

Generally, a single keying event can be used to synchronize the CVR to the FDR to within \pm 1 second, due to the FDR's $1 \mathrm{~Hz}$ sample rate for "Key VHF." Using multiple keying events may increase the accuracy of the synchronization. In this case, the start and end times for six radio transmissions (12 keying events) were evaluated. Based on these 12 events, the resulting equation provided below is accurate within \pm 0.1 (one tenth) of a second.

CVR Elapsed Time $+63732.8=$ FDR SRN

The time correlation from FDR SRN to Eastern Standard Time was provided by the Aircraft Performance Specialist:

Eastern Standard Time $=$ FDR SRN-9701.119 (where [EST] is expressed as seconds after midnight). (Brazy, 2009, p. 2).

The CVR to FDR time alignment problem is a specific instance of the general problem of time series analysis. Time series analysis is a method for analyzing time series data that can be divided into two segments, one of which is time-domain. Time-domain methods include convolution, auto-correlation, and cross-correlation analysis. Crosscorrelation is a mathematical process of determining how much function $f$ must be shifted along the $x$-axis to be identical to function $g$ and is a necessary step in a broad variety of applications, from forensics to chemistry (Fischer, Roth, \& Buhmann, 2007).

Gregor (2006) characterizes the CVR to FDR time alignment problem as having the following components: (a) knowing which FDR event corresponds to which CVR event; and (b) optimizing the offset between two series of events. For the purpose of discussion, the first step will be referred to as pattern matching, and the second step as alignment optimization. Each step is discussed in turn.

\section{Pattern Matching}

Gregor introduces the pattern matching problem as one of maximization of a cross-correlation between the CVR and FDR series of events (Gregor, 2006, p. 6)

$$
Z(n)=\Sigma_{\mathrm{k}} F D R(k) * C V R(k+n)
$$

where k represents an index to each of the FDR events. The cross-correlation essentially tries every combination of FDR event and CVR event, finding a maximization of the two alignment areas. In this case, each $F D R(k)$ and $C V R(k)$ is a binary value representing a one second sampling interval, however the equation can be computationally applied to real numbers. Cross-correlation can also be found in matrix operations by the name of convolution (MathWorks, 2011) as well as in the cross join operator of structured query languages (Microsoft, 2011) or as a Cartesian Product.

Linear programming (LP) can be applied to pattern matching. For example, in the case of searches being issued to multiple Internet search engines, LP was used to determine which document had the maximum relevancy across all search engine results. Amin and Emrouznejad (2010) outlined an LP model with an objective function of $\max \Sigma_{j}\left(\lambda_{k j} \omega_{j}\right)$

where $\lambda$ represents the $k^{\text {th }}$ document being returned as the $j^{\text {th }}$ ranked place and $\omega$ is the weighting being sought for the $j^{\text {th }}$ place. The associated constraints are

$$
\begin{aligned}
& \Sigma_{j}\left(\lambda_{i j} \omega_{j}\right)<=1(\mathrm{i}=1, \ldots, \mathrm{r}) \\
& \omega_{j}-\omega_{j+1}>=\varepsilon(\mathrm{j}=1, \ldots, 1-1) \\
& \omega_{1}>=\varepsilon
\end{aligned}
$$

where the first constraint bounds the relevancy of each document. The second and third constraints provide that the weights assigned to document $j$ as the progression continues from document $j$ to document $j+1$.

Insight into the pattern matching problem can also be drawn from the methods used in time series analysis and forecasting. Patterns in time series data are known to follow particular patterns, such as random, trend, seasonal, cyclical. Anderson et al. (2011) show how data can be curve-fit into various time series models for the purpose of forecasting; providing examples of how this is done in 
Microsoft Excel and LINGO. Time series forecasting may be adaptable to CVR/FDR pattern matching by curvefitting the CVR data series to the FDR.

\section{Alignment Optimization}

The alignment optimization step may be a simple offset, $\mathrm{C}$, given by

$$
t_{\mathrm{cvr}}=t_{\mathrm{fdr}}+\mathrm{C}
$$

This optimization is applicable when the timebase rate of the CVR and FDR are the same (Gregor, 2006, p. 5). If the timebase rate is different between the two samplings, such as may occur in legacy tape based units or where solid state units have flaws, a first order linear offset is appropriate, represented by

$$
t_{\mathrm{cvr}}=b^{*} t_{\mathrm{fdr}}+\mathrm{C}
$$

where $b$ represents the timebase rate difference between the CVR and FDR (Gregor, 2006, p. 5). This problem is solved through a least squares regression. The alignment optimization problem can grow more complex when one considers variations in $b$, for example when the device timebase varies due to mechanical reasons, power fluctuations, or software anomalies.

\section{Research Methodology}

The general problem is the synchronization of one or more series of events, recorded at different sample rates, with different areas of emphasis and importance, with different tolerances, $r$, in the sampling timebase, and a different number of events in the timelines. The problem is delimitated for this research to consider the synchronization of two series of events, recorded at different sampling rates, all of equal importance, where the timebase tolerance, $r$ is assumed to be $r=0$. The $r=0$ assumption means, for example, that 8,000 seconds in the CVR must be 8,000 seconds in the FDR: there is no allowance for different timebase rates within the series of events. One timeline represents CVR microphone keyings recorded in relative time (i.e., the start of the timeline is 0 seconds), and another timeline represents FDR microphone keyings, represented in clock time, or Greenwich Mean Time (GMT). CVR data will be expressed in elapsed seconds and FDR data expressed as seconds after midnight. For simplicity, FDR sample data will be formed such that no GMT times cross midnight, i.e., all times will be greater than zero.

A test-driven development (TDD) approach was used to develop the initial theoretical model. Kent Beck, author of Extreme Programming Explained (2000), defines TDD as an approach to software development where tests are written before code. With an appropriate number of purposively created test scenarios, when all the scenarios create the intended results, the coding effort is completed (Beck, 2000). Following the spirit of a TDD approach, many test cases were written before creating the final model and additional test cases were written to further exercise and explore the model as experience was gained from prior runs.

The alignment of the CVR and FDR recordings is accomplished in two steps: matching and optimization. Matching involves aligning the CVR and FDR time series where a microphone keying on the CVR is matched with the identical microphone keying on the FDR. Once microphone keying events have been matched, the exact alignment of each time series is optimized. The second optimization step is necessary because, as will be shown, the matching step only tries a discrete number of trial offsets to align the two series of events; optimization is necessary to try a continuum of offsets.

\section{Step 1 - Matching}

In step 1, two series of events are matched. Specifically, the first series consists of start and stop times for CVR radio transmissions. The second series consists of start and stop times from an FDR sampling based on microphone keyings. It is assumed that both series are a sampling of the same set of events; however, each series has a different time reference and different sampling accuracy. As shown in Figure 1, the intent is to shift the time frames until the patterns of events are in phase, i.e., aligned.

During the course of TDD, it was discovered that pretreatment of the FDR start and end times was necessary to properly execute both step 1 and step 2 processing. Figure 2 shows the nature of FDR sampling. Since the FDR only samples once per second, the transmission represented in Figure 2 by samples 2 and 3, may have begun anywhere after sample 1 and ended anywhere before sample 4. As such, the model allows for input of the FDR one second sampling rate and creates adjusted FDR start and end times. The adjusted start time is created by subtracting .999 seconds from the provided start time; the adjusted end time is created by adding .999 seconds to the provided end time. This constant is added or subtracted because the transmission could have occurred at any time within the one second. As the transmission start or end occurred at some point within the recorded second, to determine the maximum range where the transmission occurred, the .999 seconds expands the window to the most extreme possibility. This creates two series for the FDR, represented as $r F D R$ and $a \mathrm{FDR}$, raw and adjusted, respectively, as shown in Figure 2. These times provide

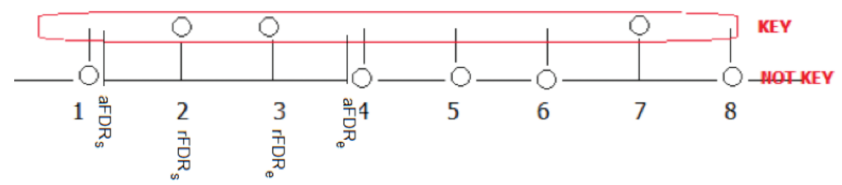

Figure 2. FDR sampling example, where "Key" indicates the microphone is transmitting. 
necessary degrees of freedom in the mathematical model that were discovered during the TDD development process.

The method used to perform the alignment is as follows. Given a series of $\mathrm{CVR}$ radio transmissions, $\left(\mathrm{CVR}_{s}\right.$, $\left.\mathrm{CVR}_{e}\right)_{i \epsilon m}$ and FDR Microphone keyings, $\left(\mathrm{aFDR}_{s}\right.$, $\left.\mathrm{aFDR}_{e}\right)_{j \epsilon n}$, where $\mathrm{s}$ and e indicate start and end times, the two series are cross joined to create $m \times n$ trial displacements, $\left[S_{i \epsilon m, j \epsilon n}\right]$, according to

$$
\left[\begin{array}{ll}
\overline{C V R_{1}} & \overline{C V R_{2}} \ldots \overline{C V R_{i}}
\end{array}\right]\left[\begin{array}{c}
a \overline{F D R_{1}} \\
a \overline{F D R_{2}} \\
\vdots \\
a \overline{F D R_{J}}
\end{array}\right]
$$

where $\overline{C V R}$ and $\overline{a F D R}$ represent the midpoint of the transmission, $s+(e-s) / 2.0$ and the mathematical operation performed on each cross join is simply, $\overline{a F D R}-\overline{C V R}$.

The array of trial displacements are used in a mixed binary, linear programming model, consisting of $m \times n$ binary decision variables, $\operatorname{SMatch}_{i j}$ (where $(i, j) \in m, n$ ) with one constraint such that one and only one $S_{M a t c h}$ can be 1. The objective function is designed to maximize the overlap of the CVR and FDR transmissions and is expressed as

$\operatorname{Max}$

$\sum_{(i, j) \in(m, n)}\left(\sum_{k \in m} \sum_{l \in n}\left\{f_{\text {area }}\left(S_{i, j}, C V R_{k}, a F D R_{l}\right)\right\}\right)$

* $\mathrm{SMatch}_{i, j}$

where the $f_{\text {area }}$ is a custom function to calculate the pseudoarea of overlap for each of the conditions expressed in Figure 3. For example, the pseudo-area of overlap for case 4 from Figure 3 is defined by

$$
\begin{aligned}
& \left(a F D R_{s(l)}-\left(S_{i, j}+C V R_{e(k)}\right)\right)^{2}- \\
& \left(\left(S_{i, j}+C V R_{s(k)}\right)-a F D R_{e(l)}\right)^{2}
\end{aligned}
$$

with other overlap areas likewise calculated (not shown).

The only constraint is on the binary SMatch,

$$
\sum_{(i, j) \in(m, n)}\left(\text { SMatch }_{i, j}=1\right)
$$

The result of the mixed binary, linear program is an offset, $S$, which is the one $S$ out of all the $m \times n S^{\prime}$ s that maximizes the total area of overlap of the two series of transmissions. This result is then used in a dynamic programming loop to create the aligned transmissions in a format suitable for the Step 2-Optimization. The pseudocode for the loop is shown in Listing 1.

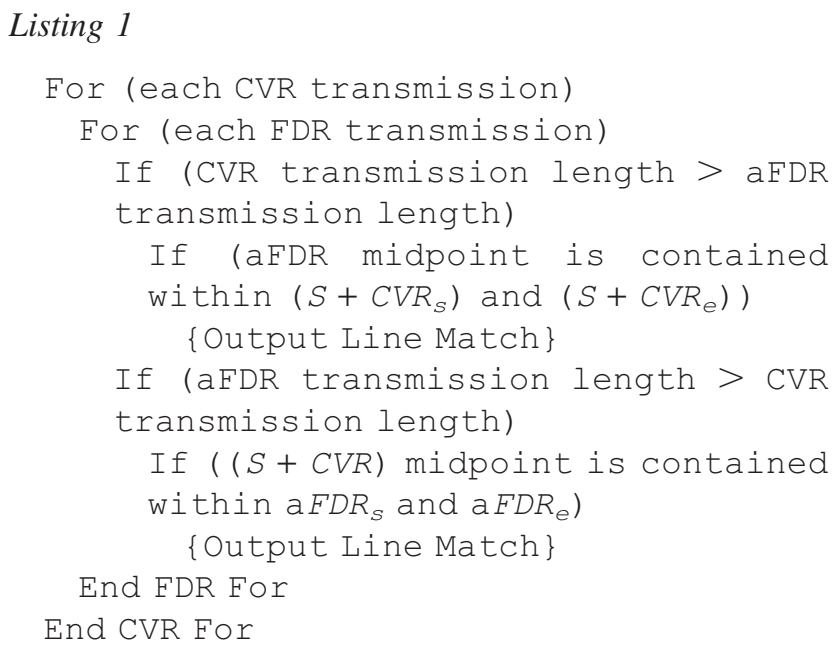

Each output line of the matching step consists of six numbers, as shown in Listing 2. The CVR times in Listing 2 are the CVR times provided as input to the step 1 model. The FDR outputs are both the adjusted FDR times and the raw FDR times (the FDR times provided as input to the model).

\section{Listing 2}

\section{$\mathrm{CVR}_{s} \mathrm{CVR}_{e} \mathrm{aFDR}_{s} \mathrm{aFDR}_{e} \mathrm{rFDR}_{s} \mathrm{rFDR}_{e}$}

The dynamic programming step also lists those points that did not find a matching CVR or FDR point. This can happen if the CVR analyst did not hear or code a transmission, or if the FDR did not record a transmission, which can occur for transmissions less than the one second sample rate.

The test cases used and outcomes observed are summarized in Table 1, all of which are included in the online Appendix. In Table 1, reference is made to a perturbed sets of events. This is expanded on in the section

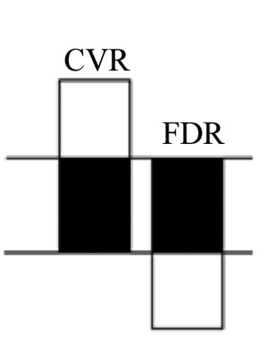

Case 1

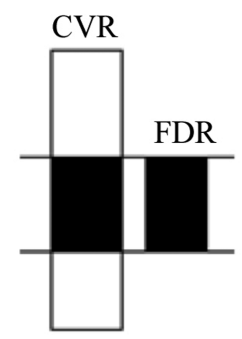

Case 2

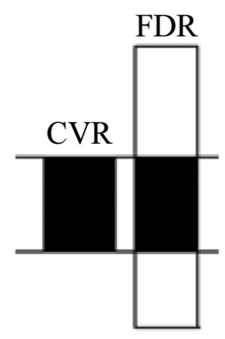

Case 3

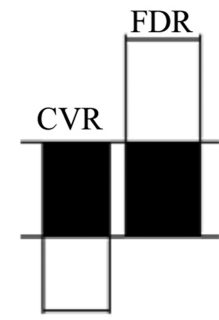

Case 4

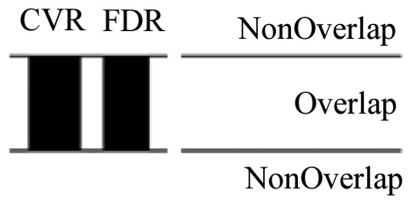

Case 5

Figure 3. Permutations of overlap of CVR and FDR events. 
Table 1

Step 1 - Matching Test Cases and Summary

\begin{tabular}{|c|c|c|}
\hline Case & Description & Outcome \\
\hline 1 & 7 perfectly aligned events. Expected outcome: match all. & All events matched. \\
\hline 2 & Same as case 1, with one missing CVR event. Expected outcome: 6 events match. & 6 events matched. \\
\hline 3 & $\begin{array}{l}6 \text { randomly perturbed CVR events, and } 7 \text { FDR events (one CVR event missing). Known to } \\
\text { fail step 2. Expected outcome: } 6 \text { events match. }\end{array}$ & 6 events matched. \\
\hline 4 & 3 events, non-unique in pattern. Expected outcome: random match. & $\begin{array}{l}\text { Matched on first event, no warning of } \\
\text { non-unique match. }\end{array}$ \\
\hline 5 & 7 CVR events and 6 FDR events with no relationship in patterns. Expected outcome: no events match. & One event matched, all others failed. \\
\hline 6 & Similar to case 3. Expected outcome: all events match. & All events matched. \\
\hline 7 & $\begin{array}{l}\text { Perfectly perturbed events. Starting with one event, adds a perfectly perturbed event until a total of } \\
8 \text { events are tested. Expected outcome: all cases should match all events provided. }\end{array}$ & $\begin{array}{l}\text { Each case matched all provided } \\
\text { events. }\end{array}$ \\
\hline 8 & $\begin{array}{l}\text { Multiple cases: } 8 \text { total events. Each sub-case removed a random event from CVR and/or FDR event } \\
\text { list. Expected outcome: all cases should match all events provided. }\end{array}$ & $\begin{array}{l}\text { Each case matched all provided } \\
\text { events. }\end{array}$ \\
\hline 9 & $\begin{array}{l}\text { Perfectly perturbed events, however, a linear trend of } .025 \% \text { is added to the base rate of the CVR. } \\
\text { Expected outcome: match all points, expect to fail step } 2 \text {. }\end{array}$ & All events matched. \\
\hline 10 & $\begin{array}{l}\text { Perfectly aligned events, similar to case 1, except } \mathrm{S} \text { will be negative. Expected outcome: match all } \\
\text { points, trial } \mathrm{S} \text { negative. }\end{array}$ & All events matched. \\
\hline
\end{tabular}

explaining Step 2 - Optimization; for the purpose of Step 1 - Matching, it is sufficient to explain perturbation as the introduction of a random offset greater than or equal to 0 and less than 1 to each start and end time of the CVR. All cases ultimately performed as expected, except cases 4 and 5. In case 4 , the pattern was not unique yet no warning was provided. In case 5, there was no pattern to be matched, yet one point matched. These cases are discussed further in the discussion section.

\section{Step 2 - Optimization}

The analysis could end with the first step if accuracy to one second is acceptable; however, accident investigations often require the most accurate timeline possible. To further align the recorded events, the CVR and FDR events are analyzed to determine an optimum offset. The expected input is shown in Listing 3. Each row represents the same event recorded by the CVR and FDR. The first two columns are CVR start and end times $\left(\mathrm{CVR}_{s}, \mathrm{CVR}_{e}\right)$; the next two columns are adjusted FDR start and end times (as explained in Step 1-Matching) $\left(\mathrm{aFDR}_{s}, \mathrm{aFDR}_{e}\right)$; and the last two columns are the raw, sampled FDR start and end times $\left(\mathrm{rFDR}_{s}, \mathrm{rFDR}_{e}\right)$. The properly formatted file is a product of Step 1 Matching, though the input file could also be manually created.

\section{Listing 3}

$\begin{array}{llll}1000.045 & 1000.691 & 4999.001 & 5000.999 \\ 5000.0005000 .000 & & \\ 1050.440 & 1055.666 & 5049.001 & 5055.999 \\ 5050.0005055 .000 & & \\ 1700.089 & 1720.742 & 5699.001 & 5720.999 \\ 5700.0005720 .000 & & \\ 2010.130 \quad 2012.919 & 6009.001 & 6012.999 \\ 6010.0006012 .000 & & \\ 2055.714 \quad 2056.395 & 6054.001 & 6056.999 \\ 6055.0006056 .000 & & \\ 2560.952 & 2567.627 & 6559.001 & 6567.999 \\ 6560.0006567 .000 & & \\ 3000.324 \quad 3001.379 & 6999.001 & 7001.999 \\ 7000.0007001 .000 & & \\ 3005.255 \quad 3007.646 & 7004.001 & 7007.999 \\ 7005.0007007 .000 & & \end{array}$

The relationship of the CVR event to the adjusted and raw FDR event is shown in Figure 4. The rFDR points show the 1-second samples of microphone keyings by the FDR; in Figure 4 this is shown in points 2 and 3. The aFDR points show the maximum possible extent of the microphone keyings given the FDR sample rate. The aFDR points are infinitesimally close to the adjacent sampling frame. The $\mathrm{CVR}_{s}$ point must lie somewhere between

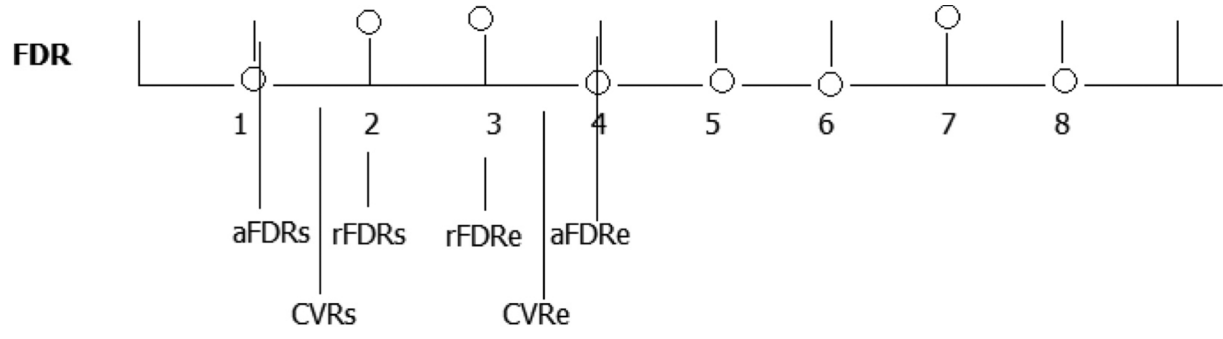

Figure 4. Relationship of CVR, aFDR, and rFDR. 
$\mathrm{aFDR}_{s}$ and $\mathrm{rFDR}_{s}$; and the $\mathrm{CVR}_{e}$ point must lie somewhere between $\operatorname{rFDR}_{e}$ and aFDRe.

In the previous section, Step 1-Matching, the term perturbed test sets was introduced, Figure 4 permits an expanded discussion of this term. A perfectly aligned CVR and FDR event is defined by $\mathrm{CVR}_{s}=\mathrm{FDR}_{s}$ and $\mathrm{CVR}_{e}=$ $\mathrm{FDR}_{e}$. This type of perfect alignment has no slack-shifting the points left or right causes infeasibility. A random perturbation transforms the perfectly aligned CVR event by $\mathrm{CVR}_{(s, e)} \pm \varepsilon$, where $\varepsilon$ is a random number greater than 0 and less than 1. This simulates a near real world scenario considering the qualitative nature of CVR transmission identification. The perfectly perturbed set is $\mathrm{CVR}_{s}-\varepsilon$ and $\mathrm{CVR}_{e}+\varepsilon$. Perfectly perturbed events represent the ideal CVRFDR alignment problem, given the constraints defined in this paper. While a perfectly perturbed and perfectly aligned set of events do have feasible solutions, a random perturbation will likely not have a feasible solution.

The point relationship shown in Figure 4 is the basis for a linear optimization model. The objective function is

$$
\begin{aligned}
& \text { Min } \\
& \sum_{i}\left|a F D R_{s}-\operatorname{align} C V R_{s}\right|+\left|a F D R_{e}-\operatorname{align} C V R_{e}\right|
\end{aligned}
$$

where alignCVR $\mathrm{s}$ and alignCVR $\mathrm{e}_{\mathrm{e}}$ are defined in the constraints,

$$
\begin{aligned}
& \operatorname{align} C V R_{s}=S+C V R_{S} \\
& \operatorname{align} C V R_{e}=S+C V R_{e}
\end{aligned}
$$

and the solution is subject to the constraints

$$
\begin{aligned}
& \text { align } C V R_{s} \geq a F D R_{s} \\
& \text { align } C V R_{s} \leq a F D R_{s} \\
& \text { align } C V R_{e} \geq r F D R_{e} \\
& \text { align } C V R_{e} \leq a F D R_{e}
\end{aligned}
$$

where $\mathrm{S}$ is the CVR offset being sought.

Equations 5 through 7 create a set of linear programming equations, similar in form to a linear regression (Anderson et al., 2011). The addition of equations 8 through 11 provide the constraints needed to optimize the CVR/FDR alignment model consistent with Figure 4.

The answer produced in Step 2-Optimization, while an optimum solution, will have slack in the solution consistent with the nature of the FDR sampling rate and optimization models (Anderson et al., 2011). While sensitivity analysis may offer insight into the slack in the constraints, a more user-friendly, automated approach can be had by running a relaxed solution two additional times. The first time, Equation 5 is modified to

\section{$\operatorname{Min} S$}

and the second time, Equation 5 is modified to

$$
\operatorname{Max} S
$$

The additional two runs push the solution towards a feasible, constrained solution either towards the left or right, as shown in Figure 5. The terms left and right are used metaphorically for explanation purposes consistent with Figure 5. The two additional solutions produce two additional displacements, $S$, for a total of three displacements. The two additional displacements represent the extreme limits of all feasible solutions, with the solution containing Equation 5's objective function providing a balanced offset considering both start and end times.

The output of Step 2 - Optimization is a three-part solution with an optimum offset, $S$, expressed in the context of a sentence dynamically concatenated from the execution, such as, "CVR elapsed time was aligned to FDR elapsed time by means of a linear programming model. 8 transmissions from the CVR were aligned with 8 identical transmissions from the FDR. The resulting 17-decision variable, 48-constraint linear programming model was solved resulting in an offset of 4000.042 seconds \pm 0.117 seconds". In the exemplar sentence, all numbers are dynamically calculated and replaced in the equation. The output also contains a listing of the aligned events, as shown in Listing 4. Additionally, a number of charts are produced, including a display of slack in the solution, an example is shown in Figure 6.

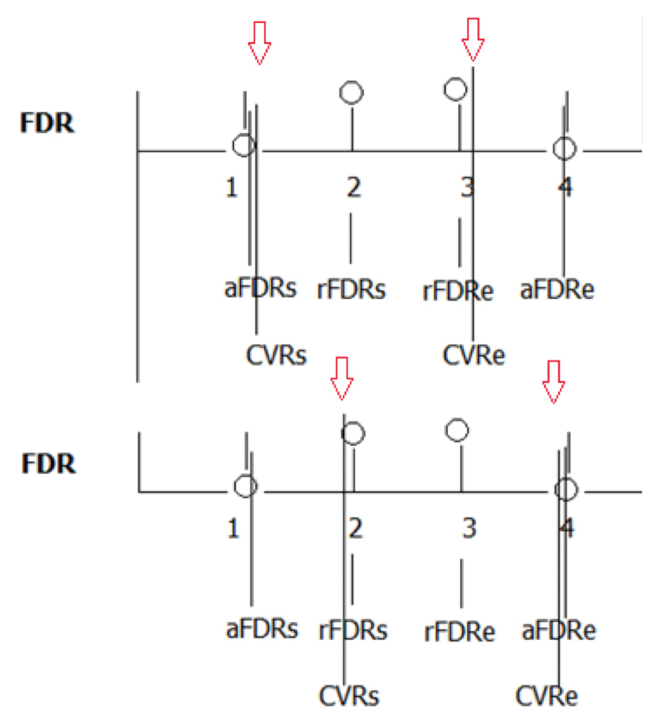

Figure 5. Top time scale shows solution pushed towards the left and bottom time scale shows solution pushed towards the right. 


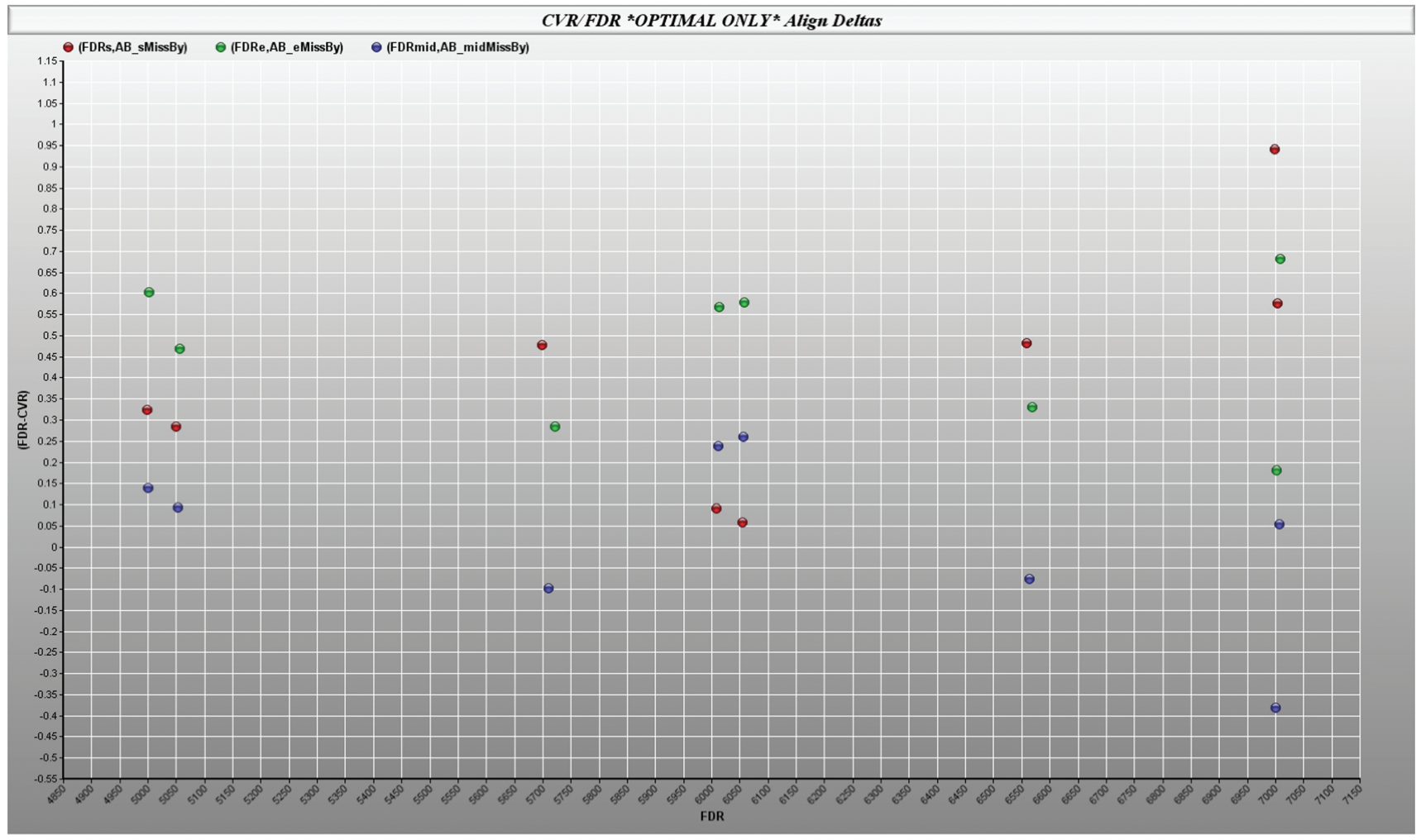

Figure 6. CVR alignment solution slack per event.

\section{Listing 4}

\section{FEASIBLE STATUS: 6}

AB_S $=4000.042$,with Start/End Relaxations: 0.000/ 0.000

s cvr 4999.325 s fdr 4999.001 (delta s) +0.324 e cvr

5000.396 e fdr 5000.999 (delta e) +0.603

s cvr 5049.285 s fdr 5049.001 (delta s) +0.284 e cvr

5055.529 e fdr 5055.999 (delta e) +0.470

s cvr 5699.479 s fdr 5699.001 (delta s) +0.477 e cvr

5720.714 e fdr 5720.999 (delta e) +0.285

s cvr 6009.092 s fdr 6009.001 (delta s) +0.091 e cvr 6012.431 e fdr 6012.999 (delta e) +0.568

s cvr 6054.059 s fdr 6054.001 (delta s) +0.058 e cvr 6056.420 e fdr 6056.999 (delta e) +0.579

s cvr 6559.482 s fdr 6559.001 (delta s) +0.481 e cvr 6567.667 e fdr 6567.999 (delta e) +0.332

s cvr 6999.941 s fdr 6999.001 (delta s) +0.940 e cvr 7001.818 e fdr 7001.999 (delta e) +0.181

s cvr 7004.577 s fdr 7004.001 (delta s) +0.576 e cvr 7007.316 e fdr 7007.999 (delta e) +0.683

The test cases run in Step 1 - Matching were used as inputs to Step 2 - Optimization; Table 2 summarizes the test cases. Cases 1 and 2 are perfect alignment cases and produce an offset with no slack. Case 3 has random perturbations that were purposively contrived to create an infeasible solution, by adding $\varepsilon$ to both $\mathrm{CVR}_{\mathrm{s}}$ and $\mathrm{CVR}_{\mathrm{e}}$. As expected, Case 3 was infeasible. While Case 4 is problematic, in that Step 1 found a match even though the pattern of events was not unique, the Step 2-Optimization found an offset with no slack, as expected; this case is addressed further in the discussion section. Case 5 and 6 were infeasible as expected. The multiple runs in Case 7 all had feasible solutions, with slack decreasing as more events were added. Case 8 was similar to Case 7, however events were randomly removed from the CVR and FDR lists and still all solutions were feasible as expected. Case 9 presented a set of events where the timebase of the CVR was other than one; this simulates the case were the CVR was either recorded or played back such that 1 second equaled 1.00125 seconds. Given the total duration from the first event to the last event was over 4000 seconds, the drift in events was such that an infeasible outcome was created. Case 9 is addressed in the discussion.

\section{Discussion}

Through TDD and linear programming, a model has been created to align and optimize CVR and FDR timelines. The process is completed in two steps, first matching the events between recordings and then optimizing the alignment.

\section{Step 1 - Matching}

Step 1 presented a robust ability to match events. While robust, Step 1 requires analyst review to ensure the solution produced is in fact unique since there is a possibility that a 
Table 2

Step 2 Optimization Test Cases and Summary

\begin{tabular}{|c|c|c|}
\hline Case & Description & Outcome \\
\hline 1 & 7 perfectly aligned events. Expected outcome: feasible, no slack. & Feasible solution; $S=4000 \pm 0$. \\
\hline 2 & Same as case 1, with one missing CVR event. Expected outcome: feasible, no slack. & Feasible solution; $\mathrm{S}=4000 \pm 0$. \\
\hline 3 & $\begin{array}{l}6 \text { randomly perturbed CVR events, and } 7 \text { FDR events (one CVR event missing). All CVR events } \\
\text { have perturbations added. Expected outcome: infeasible solution. }\end{array}$ & Infeasible solution. \\
\hline 4 & 3 events, non-unique in pattern. Expected outcome: feasible, no slack. & Feasible solution; $\mathrm{S}=4000 \pm 0$. \\
\hline 5 & $\begin{array}{l}7 \text { CVR events and } 6 \text { FDR events with no relationship in patterns. Expected outcome: } \\
\text { infeasible solution. }\end{array}$ & Infeasible solution. \\
\hline 6 & Similar to case 3 . Expected outcome: infeasible solution. & Infeasible solution. \\
\hline 7 & $\begin{array}{l}\text { Perfectly perturbed events. Starting with one event, adds a perfectly perturbed event until a total of } \\
8 \text { events are tested. Expected outcome: all solutions feasible, with decreasing slack per case. }\end{array}$ & $\begin{array}{l}\text { Feasible solution of decreasing } \\
\text { slack. See graph in Figure } 8 \text {. }\end{array}$ \\
\hline 8 & $\begin{array}{l}\text { Multiple cases: } 8 \text { total events. Each sub-case removed a random event from CVR and/or FDR event } \\
\text { list. Expected outcome: feasible solutions with less slack as more points. }\end{array}$ & $\begin{array}{l}\text { Feasible solution with less slack } \\
\text { with more cases. }\end{array}$ \\
\hline 9 & $\begin{array}{l}\text { Perfectly perturbed events, however, a linear trend of } .125 \% \text { is added to the base rate of the CVR. } \\
\text { Expected outcome: infeasible solution. }\end{array}$ & Infeasible solution. \\
\hline 10 & $\begin{array}{l}\text { Perfectly aligned events, similar to case 1, except } \mathrm{S} \text { will be negative. Expected outcome: } \\
\text { match all points, trial } \mathrm{S} \text { negative. }\end{array}$ & Feasible solution, no slack, $\mathrm{S}<0$. \\
\hline
\end{tabular}

non-unique pattern of events may exist between the CVR and FDR. The analyst can use Step 1 as a tool to quickly discover a likely match, and then review other parts of the CVR to verify event alignment, such as crew callouts related to altitude, heading, and airspeed, or aural alerts all of which may have corresponding FDR events.

\section{Step 2 - Optimization}

Step 2 presented solutions with quantitative, nonprobabilistic measure of slack within feasibility constraints. All the infeasible solutions from Table 2 can be explained by Figure 7 . When CVR perturbations create a scenario where the CVR timeline recorded a radio transmission yet the FDR did not sample a microphone keying, an infeasible situation occurs; this is shown by $\mathrm{CVR}_{s}$ in Figure 7. One of the ways the scenario in Figure 7 can occur was shown in Case 9 of Table 2: a timebase of other than 1 in the CVR recording/playback. This can occur in tape or solid-state units and is in fact part of the regulatory timebase tolerance limit of $.125 \%$ per hour. The scope of this research effort limited the defined problem to a timebase of 1 ; however, the next section discusses model modifications.

It is intuitively expected that more events lead to a more optimal alignment between CVR and FDR - a solution with less slack. The multiple sub-cases of Case 8 from Table 2

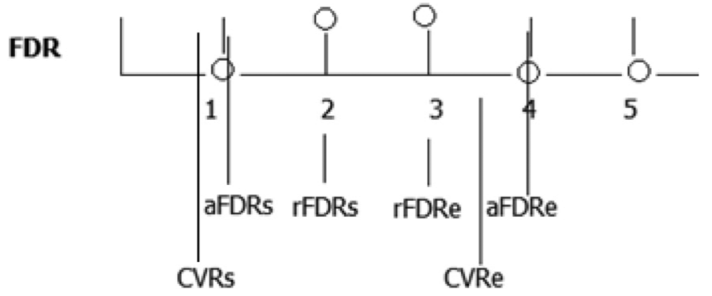

Figure 7. Example of infeasible solution. The CVRs has been recorded in a location where the sampling showed no Microphone keying. were used to plot the improved alignment achieved through additional points and is shown in Figure 8. Each optimization solution creates three values of the offset, $S$ : one pushed to the left limit of feasibility, one to the right, and one balanced between left and right. Figure 8 plots $S$ for each of these three solutions, along with the maximum absolute value difference between $S$ expressed as Bounds. The plot shows that the addition of only a few points quickly causes an asymptotic convergence of the bounds of $S$.

\section{Theoretical and Practical Implications}

Before the dawn of solid state recorders, timebase variation was an ecological reality. However, solid-state recorders present the possibility for near-negligible timebase variations, even below the regulatory $.125 \%$ per hour. The benefit of the model presented herein is that it allows for discovery of an optimum solution with a timebase tolerance $r=0$. As a "first pass" solution to CVR/FDR alignment, if the solution presented herein is discovered to be feasible then a minimal timebase error can be inferred with the benefit of producing error bounds based on feasibility constraints.

If an infeasible solution is discovered, then sources of error can be investigated such as analyst perception of CVR radio transmissions or FDR malfunctions. Once these other confounding factors are eliminated as possibilities, timebase variations can be further investigated. If the timebase is found to be suspect, then a first or higher order linear regression or piecewise spline can be used to fit the CVR to the FDR events.

Real world considerations of CVR and FDR forensic analysis introduce challenges that influence the use of the model. The data formats of the CVR and FDR events are qualitatively and quantitatively different. CVR events are determined by analyst audio review of recorded information 


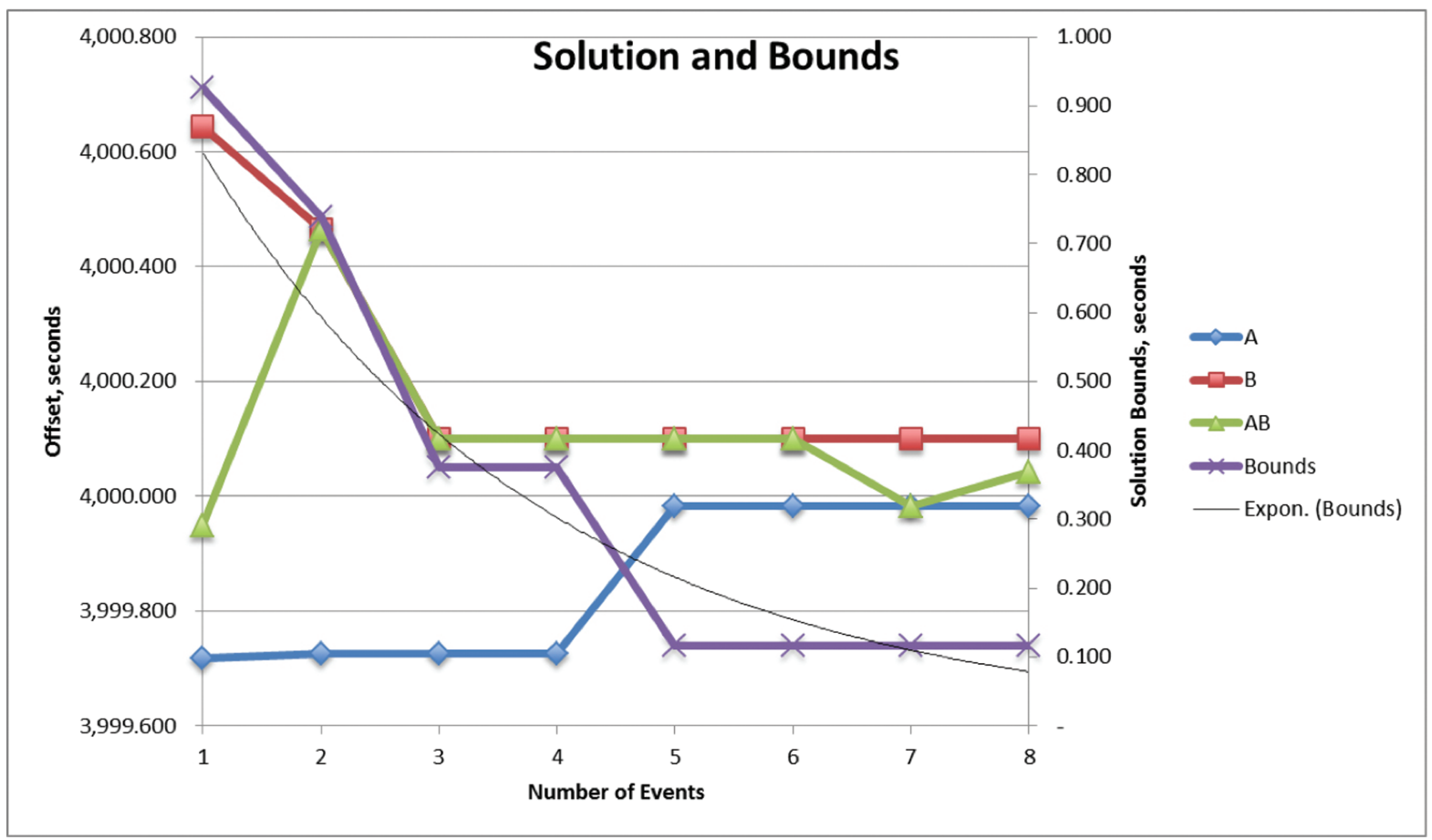

Figure 8. Benefit of additional points on solution.

aided by visual representations of the waveform. This is an inherently qualitative exercise. Further, the CVR events are inherently event orientated - a radio transmission has a start and end time. Conversely, FDR events are inherently quantitative and stateless. The FDR does not directly record radio transmissions, rather it samples when a microphone keying is in the transmit or non-transmit state.

The different sources of the CVR and FDR data present a data conversion challenge. FDR Microphone keying data is a series of bits recorded every second in FDR relative units. CVR transmissions are start and stop events, either recorded in seconds or in traditional hour:minute:second format. In order to use the model presented herein, the FDR binary data must be converted into events, and the CVR data into seconds and fractions thereof; this data conversion step may require custom programming.

While LINGO was used as the programming language for this research, two part methodology is conducive to using any number of computing languages. Step 1 is fundamentally finding the maximum of the Cartesian product of two arrays. Step 2 can use the Step 1 trial alignment, $S$, as the basis of a for-next loop over a range of $S^{\prime}$ s with a small increment of 0.01 . A range of feasible and non-feasible solutions could quickly be identified over a more than adequate five second span.

Significantly, this model suggests when a feasible solution can be found; an error bound can be reported. While this is mathematically correct, it may not be ecologically correct. That is, the qualitative process of determining CVR transmit start and stop times introduces an error that cannot be accounted for by the model alone.

\section{Limitations}

This model is built on the assumption that the CVR and FDR recordings contain overlapping events. This model will produce no relevant results if a portion of either recording is destroyed and there are no overlapping events.

Further, as mentioned the model assumes $r=0$.

\section{Future Research}

The Step 2 model presented herein is essentially a zero ${ }^{\text {th }}$ order regression with constraints. The zero ${ }^{\text {th }}$ order model can be modified to a first order regression model. Equation 6 and 7 can be modified as follows

$$
\begin{aligned}
& \operatorname{align} C V R_{s}=S+b * C V R_{s} \\
& \operatorname{align} C V R_{e}=S+b * C V R_{e}
\end{aligned}
$$

The LINGO code listings in the online Appendix present the model in this fashion, however constraints are imposed to force $b=1$. As stated, by performing first-pass analysis with $b$ constrained to unity, feasible solutions can be expressed with numerical error bounds.

In addition to the first-order regression modification of Equations $6 \mathrm{a}$ and $7 \mathrm{a}$, the constraints can be relaxed to permit a feasible solution. Equations 8 through 11 can be modified

$$
\begin{aligned}
& \text { alignCVR } R_{s} \geq a F D R_{s}-\text { relaxStart } \\
& \text { align } C V R_{s} \leq r F D R_{s}+\text { relaxEnd }
\end{aligned}
$$




$$
\begin{aligned}
& \text { alignCVR } \geq r F D R_{e}+\text { relaxStart } \\
& \text { alignCVR } R_{e} \leq a F D R_{e}-\text { relaxEnd }
\end{aligned}
$$

where relaxStart and relaxEnd are positive numbers set by the user.

The relaxation parameters allow for discovery of a feasible solution. The LINGO code listings in the online Appendix present the model in this fashion, with the relaxation parameters set to 0 .

\section{Conclusion}

The two step solution herein presents a method to align CVR and FDR events producing an error bounded offset result. The method was developed by using a TDD process. The optimized offset result can be stated in a consistent and automated format of the form, "CVR elapsed time was aligned to FDR elapsed time by means of a linear programming model. 8 transmissions from the CVR were aligned with 8 identical transmissions from the FDR. The resulting 17-decision variable, 48-constraint linear programming model was solved resulting in an offset of 4000.042 seconds \pm 0.117 seconds".

The model provides a first-pass, zero ${ }^{\text {th }}$ order analysis of CVR and FDR events. When this zero ${ }^{\text {th }}$ order model produces a feasible result, the analyst can gain confidence the timebase of the CVR and FDR were the same while reporting a quantifiable error bound on the offset $S$.

\section{Acknowledge}

The authors wish to acknowledge Dr. Dothang Truong for his critique and support of this paper during his ORMS class. This work is the authors' alone and does not reflect the official position of their respective employers.

\section{Appendix}

See Appendix online at: https://erau.blackboard.com/ webapps/cmsmain/webui/_xy-38557552_1-t_ggVtZ07h

\section{References}

Amin, G., \& Emrouznejad, A. (2010). Finding relevant search engines results: A minimax linear programming approach. The Journal of Operational Research Society, (61)7, 1144-1050.

Anderson, D. R., Sweeney, D. J., Williams, T. A., Camm, J. D., \& Martin, K. (2011). An introduction to management science: quantitative approaches to decision making (13th ed.). Mason, $\mathrm{OH}$ : Cengage South-Western.

Beck, K. (2000). Extreme programming explained. Boston, MA: AddisonWesley.

Brazy, D. (2009). Cockpit voice recorder group chairman's factual report, loss of thrust in both engines, US Airways flight 1549 Airbus Industries A320214, N106US. (Accident Report No. DCA09MA026). Washington, DC: National Transportation Safety Board.

Federal Aviation Administration. (2010). Airplane Flight Data Recorder Specifications. 14 Code of Federal Regulations, Part 121.

Fischer, B., Roth, V., \& Buhmann, J. (2007). Time-series alignment by non-negative multiple generalized canonical correlation analysis. BMC Bioinformatics, 8(Suppl 10), S4, doi: 10.1186/1471-2105-8S10-S4.

Gregor, J. A. (2006). A mathematical cross-correlation for time-alignment of cockpit voice recorder and flight data recorder data. Journal of Accident Investigation, 2(1), 44-53.

MathWorks. (2011). 'Convolution matrix documentation-signal processing toolbox in Matlab'. Retrieved from http://www.mathworks.com/ help/toolbox/signal/ref/convmtx.html

Microsoft. (2011). 'Using cross joins'. Retrieved from http://msdn. microsoft.com/en-us/library/ms190690.aspx

National Transportation Safety Board. (2010). Loss of thrust in both engines after encountering a flock of birds and subsequent ditching on the Hudson river US Airways flight 1549 Airbus A320-214, N106US Weehawken, New Jersey January 15, 2009. (Accident Report No. NTSB/AAR-10/03). Washington, DC: National Transportation Safety Board.

O'Callaghan, J. (2009). Aircraft performance group chairman's performance study, loss of thrust in both engines, US Airways flight 1549 Airbus Industries A320-214, N106US. (Accident Performance Study No. DCA09MA026). Washington, DC: National Transportation Safety Board.

Schueller, R. (1998). Black box. In, K. M. Krapp \& J. L. Longe (Eds) How products are made: an illustrated guide to product manufacturing (pp. 58-61). Detroit: Gale. Retrieved from http://go.galegroup.com.ezproxy. libproxy.db.erau.edu/ps/i.do?\&id=GALE\%7CCX2896700022\&v=2. $1 \& \mathrm{u}=\mathrm{embry} \& \mathrm{it}=\mathrm{r} \& \mathrm{p}=\mathrm{GVRL} \& \mathrm{sw}=\mathrm{w}$

Smith, G. (2009). Flight data recorder group chairman's factual report, loss of thrust in both engines, US Airways flight 1549 Airbus Industries A320-214, N106US. (Accident Factual Report No. DCA09MA026). Washington, DC: National Transportation Safety Board. 